\title{
A New Dressing System Which Is Waterproof, Breathable, Bacteriostatic, Low-Cost and Reduces the Number of Dressing Changes in the Primary Total Hip Arthroplasty: A Feasibility Study
}

\section{Shilong Su}

Xiangya Hospital Central South University

Da Zhong

Xiangya Hospital Central South University

Chenggong Wang

Xiangya Hospital Central South University

Fawei Gao

Xiangya Hospital Central South University

Pengfei Lei ( $\nabla$ dr_leipengfei@126.com )

Xiangya Hospital Central South University

Yihe Hu

Xiangya Hospital Central South University

Research article

Keywords: Dressing, Waterproof, Hip arthroplasty, Feasibility

Posted Date: December 18th, 2020

DOl: https://doi.org/10.21203/rs.3.rs-129847/v1

License: (c) (i) This work is licensed under a Creative Commons Attribution 4.0 International License.

Read Full License 

.

A new dressing system which is waterproof, breathable, bacteriostatic, low cost and reduces the number of dressing changes in the primary total hip arthroplasty: a feasibility study

Authors:

Shilong $\mathrm{Su}^{+}$, Department of Orthopedics, Xiangya Hospital, Central South University, No.

87 Xiangya Road, Changsha, 410008, Hunan Province, China, Email: sushilong@csu.edu.cn

Da Zhong ${ }^{+}$, Department of Orthopedics, Xiangya Hospital, Central South University, No.

87 Xiangya Road, Changsha, 410008, Hunan Province, China, Email: zhongda_med@163.com

Chenggong Wang, Department of Orthopedics, Xiangya Hospital, Central South University, No.

87 Xiangya Road, Changsha, 410008, Hunan Province, China, Email:

wangchenggong@csu.edu.cn

Fawei Gao, Department of Radiology, Xiangya Hospital, Central South University, No.

87 Xiangya Road, Changsha, 410008, Hunan Province, China, Email: yngaofawei@163.com

Pengfei Lei, Department of Orthopedics, Xiangya Hospital, Central South University, No.

87 Xiangya Road, Changsha, 410008, Hunan Province, China, Email: dr_leipengfei@126.com

Yihe Hu, Department of Orthopedics, Xiangya Hospital, Central South University, No.

87 Xiangya Road, Changsha, 410008, Hunan Province, China, Email: csuhuyihe@163.com

Shilong $\mathrm{Su}^{+}$and $\mathrm{Da} \mathrm{Zhong}^{+}$contributed equally to this work.

\section{Correspondence to:}

Dr. Pengfei Lei, Department of Orthopedics, Xiangya Hospital, Central South University, No.

87 Xiangya Road, Changsha, 410008, Hunan Province, China, Email: dr_leipengfei@126.com 
Abstract

\section{Background}

Total hip arthroplasty is one of the most successful operations for the treatment of advanced hip osteoarthritis and osteonecrosis of the femoral head. Periprosthetic joint infection (PJI) is the most serious complication of joint replacement, and postoperative wound complication is the risk factor of PJI, so it is very important to manage the surgical wound. We innovatively invented a new dressing system to reduce the occurrence of postoperative wound complications and improve the quality of life and functional recovery of patients after operation. We designed this clinical study to confirm the clinical safety and feasibility of this new dressing system.

\section{Methods}

A total of 120 patients who underwent the primary unilateral total hip arthroplasty were enrolled in the study. The data collected included the number of dressing changes and postoperative hospital stay, The Visual Analogue Scale (VAS) score, The June 2020 - Retrospectively registered,

Harris Hip Score (HHS), The Hip disability and Osteoarthritis Outcome Score (HOOS) and The Short Form-36 (SF-36), ASEPSIS score, The Stony Brook Scar Evaluation Scale (SBSES), wound complications, and satisfaction. We made a statistical analysis of the data.

Trial registration: Chinese Clinical Trial Registry, ChiCTR2000033822. Registered 13 http://www.chictr.org.cn/showproj.aspx?proj=54735 


\section{Results}

The average number of dressing changes was $0.74 \pm 0.46$, and the average postoperative hospital stay was $3.67 \pm 0.97$ days. The new dressing system calculates that the cost of a change of dressing is 33 dollars. The results of VAS, HHS, HOOS, and SF-36 showed that with the implementation of joint replacement and the extension of recovery time, the pain, functional activity, and quality of life of patients were continuously improved. The results of the four indexes of the ASEPSIS score were all 0 . The SBSES score was $3.55 \pm 0.61$ at seven days after operation and $4.38 \pm 0.71$ at one month after operation. No wound complications were recorded until one month after the operation. One month after the operation, the satisfaction rate was $92.53 \pm 3.62 \%$.

\section{Conclusion}

We have invented such a new dressing system for surgical wounds after total hip arthroplasty and confirmed the clinical safety and feasibility of the new dressing system.

Keywords: Dressing, Waterproof, Hip arthroplasty, Feasibility

\section{Introduction}

Total hip arthroplasty is one of the most common orthopedic operations at present, and with the aging of the population, the incidence is increasing every year. It is reported that among patients undergoing total hip arthroplasty, the incidence of 
periprosthetic joint infection (PJI) is between $0.59 \%$ and $2 \%(1,2)$. As the number of joint replacements increases over time, so does the number of PJI $(3,4)$. PJI is the most serious complication of joint replacement, and it is also the main reason for the failure of primary and revision hip arthroplasty(5). Because its successful treatment requires a complex and long treatment process, it can cause significant losses to patients, hospitals, and the health care system physically, emotionally, and economically(6). Risk factors for PJI are reported in the literature, including advanced age, malnutrition, obesity, diabetes, smoking, superficial wound complications, etc(7, 8). These risk factors include superficial wound complications, indicating that proper wound care is essential for the prevention of PJI. At present, the traditional dressings of aseptic gauze and plastic tape are placed after orthopedic surgery in our hospital. In some cases, we have observed wound complications such as erythema and blisters, resulting in an increased risk of wound pain and infection. It can be seen that the traditional gauze dressing is not an ideal dressing for hip arthroplasty.

Calcium alginate dressing, as a new type of wound dressing, has a fast and strong ability to absorb exudate, which can absorb 17-20 times of its weight, and can effectively control exudation, thus prolonging the dressing change time (9). To form a gel and keep the wound moist, studies have shown that moist wounds heal faster and have less pain(10). It can also release calcium ions to promote hemostasis and inhibit bacterial growth(11). It is a new dressing with good application prospects. However, at the present stage, calcium alginate dressings are often used in combination with 
gauze dressings, which cannot overcome the shortcomings of gauze dressings, but also limit the advantages of calcium alginate dressings, such as prolonging the time of dressing change.

To solve this clinical problem, we creatively apply IV3000 film and calcium alginate dressing to the surgical incision management of patients undergoing hip arthroplasty. IV3000 film is a kind of dressing film for intravenous catheterization, with high moisture permeability(12), good waterproof performance, inhibition of bacterial colonization (13), good skin adhesion, no friction with the skin, and almost no pain removal(14). The combined use of the two not only makes use of the advantages of calcium alginate dressing in promoting incision healing and absorbing incision exudate, but also makes use of the characteristics of IV3000 film, such as breathable and waterproof, good skin adhesion, and skin-friendly type, and invents a new dressing system which is waterproof, breathable and almost free of dressing change. At the same time, this dressing system is low in cost and suitable for popularization and use in a large area.

We designed this clinical trial to confirm the clinical safety and feasibility of this new dressing system. The trial was evaluated by recording the number of postoperative dressing changes, postoperative hospitalization days and medical costs, wound complications and healing, functional recovery and quality of life of patients, selfevaluation of satisfaction. 


\section{Patients and methods}

113 The Medical Ethics Committee of Xiangya Hospital of Central South University

114 approved the research plan and we obtained the written informed consent of all

115 patients. The study was registered at www.chictr.org.cn (ChiCTR2000033822).

116 The inclusion criteria of patients are as follows: 1. Age 18 to 85 years old, 2.

117 According to physical examination and imaging data, osteoarthritis and osteonecrosis

118 of the femoral head were diagnosed. 3. It is planned to undergo the primary unilateral

119 total hip arthroplasty. Patients who have had joint surgery on any hip joint, have

120 obvious scars on any hip joint, suffer from skin diseases such as psoriasis, eczema, or

121 dermatitis and cannot complete regular follow-up should be removed.

122 From April 9, 2019, to December 20, 2019, a total of 120 patients were enrolled in

123 the study. There were 59 males and 61 females, with a median age of $57.17 \pm 12.86$

124 years old (range 21-75 years old), 62 on the left, and 58 on the right. All the

125 operations were performed by an experienced joint surgeon. The operation was

126 performed by standard posterolateral approach and the prostheses were all biological.

\section{Application of New dressing system}

129 Prophylactic antibiotic cefoxitin was routinely used in 30min before the operation.

130 The standard three-layer continuous suture method was used in all patients during the

131 operation. The articular capsule was sutured continuously with 2\# absorbable knot-

132 free unidirectional barb suture (Quill, Surgical Specialties Corporation, New York, 
USA), subcutaneous tissue was sutured with 0\# absorbable knot-free bi-directional barb suture (Quill, Surgical Specialties Corporation, New York, USA), and intradermal was sutured with 3-0 absorbable knot-free bi-directional barb suture (Quill, Surgical Specialties Corporation, New York, USA). The usage of the new dressing system: 1 . After the surgical incision was sutured, the skin of 10CM around the incision was thoroughly deiodinated with $75 \%$ alcohol (Figure $1 \mathrm{~A}) .2$. The calcium alginate dressing (Algisite $\mathrm{M}$, Smith \& Nephew, London, UK) is folded into three layers in the direction of the long axis and properly cut to a length range slightly longer than the surgical incision $1 \mathrm{~cm}$ at both ends(Figure 1B,C). 3. According to the length of the incision, three to four IV3000 films (Smith\&Nephew, London, UK) were selected and applied in the direction from the distal end to the proximal end of the limb. The two ends of the film were slightly longer than the incision about $4 \mathrm{~cm}$, and the latter film was overlapped and the previous one was about one $\mathrm{cm}$ (Figure scratches, or crimps, and there is no need to change them after discharge. Patients can take a normal bath after operation according to their living habits(Figure 1I). 


\section{Data collection}

The data we collected included four parts: the number of dressing changes and postoperative hospital stays, pain, function, and quality of life scores, wound scores and complications, and satisfaction.

\section{The number of dressing changes and postoperative hospital stay}

Patients can be discharged only when they meet stringent standards, including the ability to perform independent personal care, walk at least 70 meters on crutches, get in and out of bed and get up from chairs, and are managed with oral pain relief(15).

The postoperative hospital stay is calculated as the whole day, and the part less than one day is calculated as one day. After discharge, the patient will be assigned to a chat group to take photos and upload and evaluate the dressing under the guidance of the medical staff. All patients were not covered with dressing seven days after the operation, and the wound was wiped with $75 \%$ alcohol for three days, and the total number of dressing changes was recorded. We record the medical expenses incurred by patients using the new dressings to understand the average cost of the new dressings throughout the treatment cycle.

\section{Pain and function, quality of life score}

175 We used the Visual Analogue Scale (VAS) score, The Harris Hip Score (HHS), The 
177 (SF-36) to record the pain, function, and quality of life of patients, and to evaluate the 178 changes of perioperative patients. VAS score(16) is a one-dimensional measurement 179 of pain intensity, which is widely used in different adult populations. We used the 180 VAS score to record pain, and the VAS score was a horizontal line of fixed length, $181100 \mathrm{~mm}$. The end is defined as the limit of pain to be measured, from left (0) to right 182 (10). HHS(17) was developed to evaluate the results of hip surgery and to evaluate various hip disabilities and treatments in the adult population. It assesses pain,

184 function, deformity, and range of activity and each project has a unique digital scale.

185 The highest score for HHS is 100 . The higher the HHS, the less the dysfunction.

186 HOOS(18) is adapted from The Knee injury and Osteoarthritis Outcome Score

187 (KOOS) to assess symptoms and functional limitations associated with the hip joint.

188 The HOOS consists of 40 items that assess five independent patient-related

189 dimensions: pain, symptoms, activities of daily living (ADL), function in sports and 190 recreation, and hip-related quality of life (QOL). The scores of each subscale range

191 from 0 to 100.0 indicates an extremely serious problem, 25 indicates a serious

192 problem, 50 indicates a moderate problem, 75 indicates a minor problem, and 100

193 indicates no problem. SF36 $(19,20)$ is a self-reported general health measurement

194 tool, consisting of 36 items, divided into eight aspects: physical function, role

195 physical, general health self-assessment, social function, bodily pain, vitality, mental 196 health and role emotional. Each score ranges from 0 to 100. A lower score indicates 197 more disability, and a higher score indicates less disability. Using SF-36 to evaluate the quality of life and general psychological status of patients. The time point of the 
evaluation was recorded within one week before the operation, and one month after the operation.

\section{Wound score and complications}

ASEPSIS score is a commonly used wound assessment score (21), which consists of an objective wound assessment section, a section about wound treatment, and a section about the consequences of infection. We only use the objective wound assessment part of the ASEPSIS score(22), because we only want to evaluate the clinical appearance of the wound. SBSES score(23), proposed by Singer et al in 2007, is a wound evaluation scale used to measure the cosmetic effect of a wound, including the width, height, color, residual suture marks, and overall view of the scar. The score of each index is 0 or 1 , and the total score is calculated, ranging from 0 (worst) to 5 (best). The ASEPSIS score and the SBSES score were recorded at seven days and one month after the operation. The special follow-up technicians were based on the photos taken or on-site observation records. At the same time, the wound complications of operation.

\section{Satisfaction}

We have developed a satisfaction record table to conduct a satisfaction survey. The satisfaction record table recorded patients' satisfaction with eight parameters, including their comfort with dressings, ability to take a bath, pain treatment, doctor 
visits, length of stay, number of dressing changes, hospitalization costs, and satisfaction with the overall experience, all measured in numerical terms, with a score of 0 to 10 , with a maximum score of 80 . One month after the operation, the patients filled in the records according to their real situation.

All data collection was done by a researcher who was not involved in experimental design and surgery. All quantitative data are expressed as mean \pm standard deviation. A paired t-test was used to compare the two groups. $\mathrm{P}<0.05$, the difference was statistically significant. SPSS25.0 software (SPSS, USA) was used for analysis.

\section{Results}

1. The average number of dressing changes was $0.74 \pm 0.46$, and the average postoperative hospital stay was $3.67 \pm 0.97$ days. The application of the new dressing system requires an average of 1 calcium alginate dressing and three IV3000 films, and the cost of one dressing change is calculated to be 33 dollars.

\section{Pain and function, quality of life score}

We used VAS, HHS, HOOS, and SF-36 to record the pain, function, and quality of life of the patients, and the evaluation time was set within seven week before the operation, and one month after the operation. The VAS score decreased from $5.63 \pm 1.09$ before the operation to $0.88 \pm 0.54$ one month after the operation. The HHS score increased from $70.18 \pm 7.84$ before the operation to $80.36 \pm 4.08$ one month after the operation. The pain, symptoms, activities of daily living, function in sports and 
recreation, and quality of life related to the knee joint in the HOOS score were

244 significantly improved one month after operation compared with those before

245 operation (Table 1).

246 The SF-36 score suggested that with the implementation of joint replacement surgery

247 and the application of the new dressing system, patients' pain, functional activity, and

248 quality of life are constantly improving (Table 2).

250 Table 1 score results of VAS, HHS, HOOS

\begin{tabular}{|c|c|c|c|c|}
\hline Variable & Preoperative & $\begin{array}{c}\text { One month } \\
\text { postoperatively }\end{array}$ & $t$ value & $P$ value \\
\hline VAS & $5.63 \pm 1.09$ & $0.88 \pm 0.54$ & 43.471 & $<0.001$ \\
\hline HHS & $70.18 \pm 7.84$ & $80.36 \pm 4.08$ & 12.385 & $<0.001$ \\
\hline \multicolumn{5}{|l|}{ HOOS } \\
\hline Pain & $49.18 \pm 22.79$ & $85.03 \pm 10.61$ & 16.278 & $<0.001$ \\
\hline Symptoms & $53.19 \pm 12.05$ & $86.73 \pm 6.87$ & 28.073 & $<0.001$ \\
\hline $\mathrm{ADL}$ & $40.26 \pm 9.05$ & $91.36 \pm 5.43$ & 53.422 & $<0.001$ \\
\hline \multicolumn{5}{|l|}{ Function in } \\
\hline sports and & $41.07 \pm 17.18$ & $75.29 \pm 9.75$ & 18.319 & $<0.001$ \\
\hline \multicolumn{5}{|l|}{ recreation } \\
\hline QOL & $44.53 \pm 19.35$ & $68.23 \pm 11.24$ & 11.607 & $<0.001$ \\
\hline
\end{tabular}




\begin{tabular}{lcccc}
\hline & & One month & \\
Aspect & Preoperative & t value & $P$ value \\
& & postoperatively & & \\
\hline Physical function & $29.67 \pm 14.99$ & $60.04 \pm 14.08$ & 15.984 & $<0.001$ \\
Role physical & $19.83 \pm 18.05$ & $61.46 \pm 21.47$ & 16.131 & $<0.001$ \\
General health & $31.90 \pm 9.04$ & $64.92 \pm 9.35$ & 27.631 & $<0.001$ \\
Social function & $45.94 \pm 23.49$ & $80.15 \pm 12.72$ & 13.085 & $<0.001$ \\
Bodily pain & $35.43 \pm 16.40$ & $62.60 \pm 16.42$ & 12.874 & $<0.001$ \\
Vitality & $27.58 \pm 9.96$ & $61.04 \pm 15.90$ & 21.910 & $<0.001$ \\
Mental health & $31.77 \pm 11.92$ & $66.07 \pm 12.72$ & 23.321 & $<0.001$ \\
Role emotional & $26.67 \pm 23.11$ & $70.56 \pm 23.74$ & 16.232 & $<0.001$ \\
\hline
\end{tabular}

\section{Wound score and complications}

During the use of the new dressing system, normal bathing does not affect the

dressing, and the waterproof performance is good. The results of serous discharge,

erythema, purulent discharge, and wound defect in the ASEPSIS score were all 0

259 (Table 3). The SBSES score was 3.55 \pm 0.61 at seven days after operation and

$4.38 \pm 0.71$ at one month after operation (Table 4) . The appearance of wound

261 improved gradually with the prolongation of recovery time. And no wound

262 complications were recorded until one month after the operation. The patient's wound

263 healed well and the patient described their scars as comfortable and satisfactory in appearance (Figure 2). 
266 Table 3 The score results of the ASEPSIS

\begin{tabular}{ccc}
\hline & Seven days & One month \\
& postoperatively & postoperatively \\
\hline Serous discharge & 0 & 0 \\
Erythema & 0 & 0 \\
Purulent discharge & 0 & 0 \\
Wound defect & 0 & 0 \\
\hline
\end{tabular}

Table 4 The score results of the SBSES

\begin{tabular}{lcccc}
\hline & Seven days & One month & $t$ value & $P$ value \\
& postoperatively & postoperatively & & \\
\hline Width & $0.69 \pm 0.46$ & $0.81 \pm 0.40$ & 1.923 & 0.057 \\
Height & $0.88 \pm 0.33$ & $0.90 \pm 0.30$ & 0.470 & 0.640 \\
Color & $0.06 \pm 0.24$ & $0.69 \pm 0.46$ & 12.392 & $<0.001$ \\
Residual $\quad$ suture & $1.00 \pm 0.00$ & $1.00 \pm 0.00$ & & \\
marks & & & & \\
The overall view & $0.95 \pm 0.22$ & $1.00 \pm 0.00$ & 2.283 & 0.025 \\
& $3.55 \pm 0.61$ & $4.38 \pm 0.71$ & 8.173 & $<0.001$ \\
\hline
\end{tabular}


272 One month after the operation, the satisfaction score of the patient was $73.86 \pm 2.81$,

273 the full score was 80 , and the satisfaction rate was $92.53 \pm 3.62 \%$.

\section{Discussion}

This study confirms the safety and feasibility of our original dressing system in primary total hip arthroplasty. During the follow-up to one month after the operation, 120 patients did not have any wound complications, the wound healed well and the appearance was satisfactory; the clinical operation was simple, the number of dressing changes was significantly reduced, and the burden on patients and medical staff was reduced; it was simple and portable, waterproof and bathed, which brought convenience to the life of patients after operation.

$\mathrm{PJI}$ is a serious complication of joint replacement surgery. Once it occurs, it will bring serious medical and economic burden to patients and society. Its treatment usually requires multiple revision surgeries, and the use of antibiotics for a long time does not guarantee that the infection will be eradicated. According to the literature, the complication of the surgical wound is a major risk factor for PJI, so the management of surgical wounds is very important. Different from other surgical wounds, the surgical wound of the hip joint has its particularity: first of all, the wound of hip arthroplasty may exudate more, accompanied by persistent dressing leakage.

293 Therefore, the ideal dressing should be able to handle excessive exudates while 
maintaining a barrier to prevent bacteria from entering(24). Second, since lower limb

joint replacement is usually performed in the elderly with fragile skin, there is a

higher chance of wound complications, such as blisters and skin injuries(25). Third, since these wounds are located above the joint, the dressing should be allowed to move freely and should be able to adapt to changes in the size of the wound accompanied by flexion. Fourth, because of the implanted prosthesis, any wound complications (such as blisters) that damage skin integrity should be avoided to prevent PJI(26).

Considering the particularity of the wound after hip arthroplasty, the combination of gauze and adhesive tape which is widely used in our hospital is not appropriate. First of all, the absorption effect of the exudate of gauze dressing is not good, and it is easy to soak, which increases the frequency of dressing change. on the one hand, the wound constantly changes dressing and is open to contact with the outside air, on the other hand, the exudate cannot be absorbed in time, and the probability of breeding bacteria increases. These are all risk factors for wound infection. Second, gauze dressings often adhere to the wound after wetting, causing skin damage and pain during dressing change. Third, the surface of the gauze dressing is rough and inelastic, and multi-layer coverage will cause bloated wounds. During postoperative hip movement rehabilitation exercise, this may cause obstacles, and constant friction may also cause blisters. Fourth, gauze dressings are usually not waterproof, so patients will encounter difficulties in the normal bath and skin cleaning after the operation. Failure 
to clean the skin well, especially the skin around the wound, will also increase the risk of postoperative infection.

To solve the shortcomings of so many gauze dressings, a variety of new dressings have emerged at the present stage. First of all, incision negative pressure wound therapy (NPWT) (27) has been widely used in open wounds. Recently, some scholars have applied it to closed surgical wound management, which effectively reduces the incidence of surgical incision complications in high-risk patients, but at the same time, there are unexpected blisters, and this kind of therapy is expensive and difficult to popularize. Also, Ag ion dressing(28) has been proved to be effective in promoting wound healing and preventing bacterial colonization infection, but the high cost is also a major obstacle to its wide application. In contrast, calcium alginate dressing has more prospect of popularization and application, which has the advantages of strong absorption capacity of exudate, promoting wound healing, inhibiting bacterial growth, and stopping bleeding, and its cost is more advantageous than other new dressings. alginate dressing but also increases the advantages of waterproof bathing, good fit 
dressing is 33 dollars, which is lower than that of other new dressings. Although compared with the traditional gauze dressing system, the price of one dressing change is more expensive, but from the whole postoperative wound management cycle, the significant reduction in the number of dressing changes will not bring additional costs for patients. The average number of dressing changes is 0.74 times, which significantly reduces the number of dressing changes. some scholars(29) have reported that if the dressing is not often disturbed, the risk of infection will be reduced, while the wound dressing keeps the wound near the core body temperature, which helps the healing process. In a clean wound, the incision has a regular edge, and the wound usually closes within 48 hours, and fewer dressing changes can protect

348 the wound from repeated exposure to pathogens in the surrounding air, reducing the incidence of PJI. The new dressing system is simple and portable, does not cause pain when removing dressings, has a beautiful appearance, and has elastic changes with flexion and extension during postoperative exercise, which will not hinder rehabilitation activities. More importantly, taking advantage of the waterproof and breathable properties of IV3000 film, patients can take a bath normally after the operation, which is of great significance. We know that every patient is required to prepare the skin regularly and take a bath one day before the operation, which can reduce the risk of bacterial infection in the skin around the surgical incision. Similarly, it is also important to take a bath and wash the skin after the operation, which cannot be achieved by gauze dressing but can be achieved by the new dressing system.

359 Normal bathing after the operation can not only clean the skin around the wound, 
reduce bacterial colonization, reduce the risk of wound infection, but also improve the quality of life of patients after operation, eliminate the trouble of being unable to take a bath, and improve patient satisfaction.

We used the ASEPSIS score and SBSES score to record and score the wound to evaluate wound healing and possible wound complications. Through the observation of the previous trial, we found that the wound of the patients with the new dressing system had healed completely seven days after operation. Therefore, we set the time point for the removal of the dressing as seven days after operation. The results showed that there were no wound complications one month after the operation, and the wound healed well by the objective score of the wound. The SBSES score also got a high score in the evaluation of the appearance of the wound, and the patient reported that the appearance of the wound scar was satisfactory. The satisfaction survey shows that the satisfaction rate of patients is more than $90 \%$, indicating that the new dressing system is very popular with patients.

In this study, the results confirmed the clinical safety and feasibility of the new dressing system in the wound of total hip arthroplasty and confirmed many advantages of the new dressing system, such as reducing the number of dressing changes, waterproof and breathable, bacteriostatic, low cost. If it is necessary to verify the advantages and disadvantages of the new dressing system compared with traditional gauze dressings, it needs to be confirmed by a larger sample size of clinical 
randomized controlled trials. Such clinical randomized controlled trials are already underway, and our results will be published in the next step.

\section{Conclusion}

We have invented such a new dressing system for surgical wounds after total hip arthroplasty. By combining IV3000 films and calcium alginate dressing in a specific way, we have creatively invented a dressing system that promotes incision healing, antibacterial, absorbs incision exudate, breathable and waterproof at the same time, and has good skin adhesion. The prospective feasibility study confirmed the clinical safety and feasibility of the new dressing system.

\section{List of abbreviations}

PJI: Periprosthetic joint infection

VAS: Visual Analogue Scale

HHS: Harris Hip Score

HOOS: Hip disability and Osteoarthritis Outcome Score

SF-36: Short Form-36

SBSES: Stony Brook Scar Evaluation Scale

ADL: Activities of daily living

QOL: Quality of life

NPWT: Negative pressure wound therapy 
Ethics approval and consent to participate:

405 The study design was approved by The Medical Ethics Committee of Xiangya

406 Hospital of Central South University (No.202010128) and informed consent was

407 obtained from the patient in the study.

408

409 Competing interests:

410 The authors have no competing interests.

411

412

Funding:

413 This study was supported by National Natural Sciences Foundation of China (Grant No.

41481974360 and 81902308).

415

416

\section{Author contributions:}

417 DZ, YH, and PL conceived the original ideas of this manuscript. SS, CW, and FG 418 executed the follow-up examination and materials collection. DZ, YH, and PL read the 419 examination results, participated in the surgical and medical treatment. SS prepared the 420 figures. PL and SS prepared the manuscript.

References

425 1. Bozic KJ, Ong K, Lau E, Berry DJ, Vail TP, Kurtz SM, et al. Estimating risk in 
Medicare patients with THA: an electronic risk calculator for periprosthetic joint infection and mortality. Clin Orthop Relat Res. 2013;471(2):574-83.

2. Adeli B, Parvizi J. Strategies for the prevention of periprosthetic joint infection. J Bone Joint Surg Br. 2012;94(11 Suppl A):42-6.

3. Kurtz SM, Lau E, Schmier J, Ong KL, Zhao K, Parvizi J. Infection burden for hip and knee arthroplasty in the United States. J Arthroplasty. 2008;23(7):984-91.

4. Parvizi J, Shohat N, Gehrke T. Prevention of periprosthetic joint infection: new guidelines. Bone Joint J. 2017;99-B(4 Supple B):3-10.

5. Jafari SM, Coyle C, Mortazavi SM, Sharkey PF, Parvizi J. Revision hip arthroplasty: infection is the most common cause of failure. Clin Orthop Relat Res. 2010;468(8):2046-51.

6. Kurtz SM, Lau E, Watson H, Schmier JK, Parvizi J. Economic burden of periprosthetic joint infection in the United States. J Arthroplasty. 2012;27(8 Suppl):61-5 e1.

7. Pulido L, Ghanem E, Joshi A, Purtill JJ, Parvizi J. Periprosthetic joint infection: the incidence, timing, and predisposing factors. Clin Orthop Relat Res. 2008;466(7):1710-5.

8. Della Valle C, Parvizi J, Bauer TW, Dicesare PE, Evans RP, Segreti J, et al. Diagnosis of periprosthetic joint infections of the hip and knee. J Am Acad Orthop Surg. 2010;18(12):760-70.

9. Alsharabasy AM, Moghannem SA, El-Mazny WN. Physical preparation of alginate/chitosan polyelectrolyte complexes for biomedical applications. J Biomater 
Appl. 2016;30(7):1071-9.

10. Cai J, Karam JA, Parvizi J, Smith EB, Sharkey PF. Aquacel surgical dressing reduces the rate of acute PJI following total joint arthroplasty: a case-control study. J Arthroplasty. 2014;29(6):1098-100.

11. Sood A, Granick MS, Tomaselli NL. Wound Dressings and Comparative Effectiveness Data. Adv Wound Care (New Rochelle). 2014;3(8):511-29.

12. Lin YS, Chen J, Li Q, Pan KP. Moisture vapor transmission rates of various transparent dressings at different temperatures and humidities. Chin Med J (Engl). 2009;122(8):927-30.

13. Kawamura H, Takahashi N, Takahashi M, Taketomi A. Differences in microorganism growth on various dressings used to cover injection sites: inspection of the risk of catheter-related bloodstream infections caused by Gram-negative bacilli. Surg Today. 2014;44(12):2339-44.

14. Ha NB, Chang AC, Sullivan JS, Leonello DT. Non-operative management of fingertip injuries with an intravenous dressing. J Wound Care. 2015;24(6):276, 8-9. 15. Husted H, Troelsen A, Otte KS, Kristensen BB, Holm G, Kehlet H. Fast-track surgery for bilateral total knee replacement. J Bone Joint Surg Br. 2011;93(3):351-6. 16. Aun C, Lam YM, Collett B. Evaluation of the use of visual analogue scale in Chinese patients. Pain. 1986;25(2):215-21.

17. Harris WH. Traumatic arthritis of the hip after dislocation and acetabular fractures: treatment by mold arthroplasty. An end-result study using a new method of result evaluation. J Bone Joint Surg Am. 1969;51(4):737-55. 
470

471

472

473

474

475

476

477

478

479

480

481

482

483

484

485

486

487

488

489

490

491

18. Nilsdotter AK, Lohmander LS, Klassbo M, Roos EM. Hip disability and

osteoarthritis outcome score (HOOS)--validity and responsiveness in total hip replacement. BMC Musculoskelet Disord. 2003;4:10.

19. Ware JE, Jr., Sherbourne CD. The MOS 36-item short-form health survey (SF-

36). I. Conceptual framework and item selection. Med Care. 1992;30(6):473-83.

20. Ware JE, Jr. SF-36 health survey update. Spine (Phila Pa 1976).

2000;25(24):3130-9.

21. Wilson AP, Gibbons C, Reeves BC, Hodgson B, Liu M, Plummer D, et al.

Surgical wound infection as a performance indicator: agreement of common

definitions of wound infection in 4773 patients. BMJ. 2004;329(7468):720.

22. Wilson AP, Treasure T, Sturridge MF, Gruneberg RN. A scoring method

(ASEPSIS) for postoperative wound infections for use in clinical trials of antibiotic prophylaxis. Lancet. 1986;1(8476):311-3.

23. Singer AJ, Arora B, Dagum A, Valentine S, Hollander JE. Development and validation of a novel scar evaluation scale. Plast Reconstr Surg. 2007;120(7):1892-7.

24. Hopper GP, Deakin AH, Crane EO, Clarke JV. Enhancing patient recovery

following lower limb arthroplasty with a modern wound dressing: a prospective, comparative audit. J Wound Care. 2012;21(4):200-3.

25. Bredow J, Oppermann J, Hoffmann K, Hellmich M, Wenk B, Simons M, et al.

Clinical trial to evaluate the performance of a flexible self-adherent absorbent

dressing coated with a soft silicone layer compared to a standard wound dressing after orthopedic or spinal surgery: study protocol for a randomized controlled trial. Trials. 
493 26. Gupta SK, Lee S, Moseley LG. Postoperative wound blistering: is there a link 494 with dressing usage? J Wound Care. 2002;11(7):271-3.

495 27. Keeney JA, Cook JL, Clawson SW, Aggarwal A, Stannard JP. Incisional Negative 496 Pressure Wound Therapy Devices Improve Short-Term Wound Complications, but 497 Not Long-Term Infection Rate Following Hip and Knee Arthroplasty. J Arthroplasty. $498 \quad 2019 ; 34(4): 723-8$.

499 28. Grosso MJ, Berg A, LaRussa S, Murtaugh T, Trofa DP, Geller JA. Silver500 Impregnated Occlusive Dressing Reduces Rates of Acute Periprosthetic Joint 501 Infection After Total Joint Arthroplasty. J Arthroplasty. 2017;32(3):929-32. 502 29. Gromov K, Troelsen A, Raaschou S, Sandhold H, Nielsen CS, Kehlet H, et al. 503 Tissue Adhesive for Wound Closure Reduces Immediate Postoperative Wound 504 Dressing Changes After Primary TKA: A Randomized Controlled Study in 505 Simultaneous Bilateral TKA. Clin Orthop Relat Res. 2019;477(9):2032-8. 
511 Figure 1A. The wound was sutured and deiodized. B. Folded calcium alginate dressing

512 and three IV3000 films. C. Cut the calcium alginate dressing to both ends slightly

513 longer than the incision $1 \mathrm{~cm}$. D-H. According to the length of the incision, three IV 3000

514 films were selected and applied in the order from the distal end to the proximal end of

515 the limb. The two ends of the film were slightly longer than the incision about $4 \mathrm{~cm}$, and

516 the latter film was overlapped and the previous one was about $1 \mathrm{~cm}$. There are no air

517 bubbles between the films, and the skin and stick closely to the skin. I. After the patient

518 took a bath according to his own habits, the dressing was not affected.

519

520 Figure 2A. The wound was sutured during the operation. B. Three days after

521 operation, there was no obvious ecchymosis, swelling and exudation in the wound. $\mathbf{C}$.

522 Seven days after operation, the wound healed completely. D. One month after

523 operation, the wound of the patient showed that the scar was smooth, and the overall

524 appearance was satisfactory. 


\section{Figures}
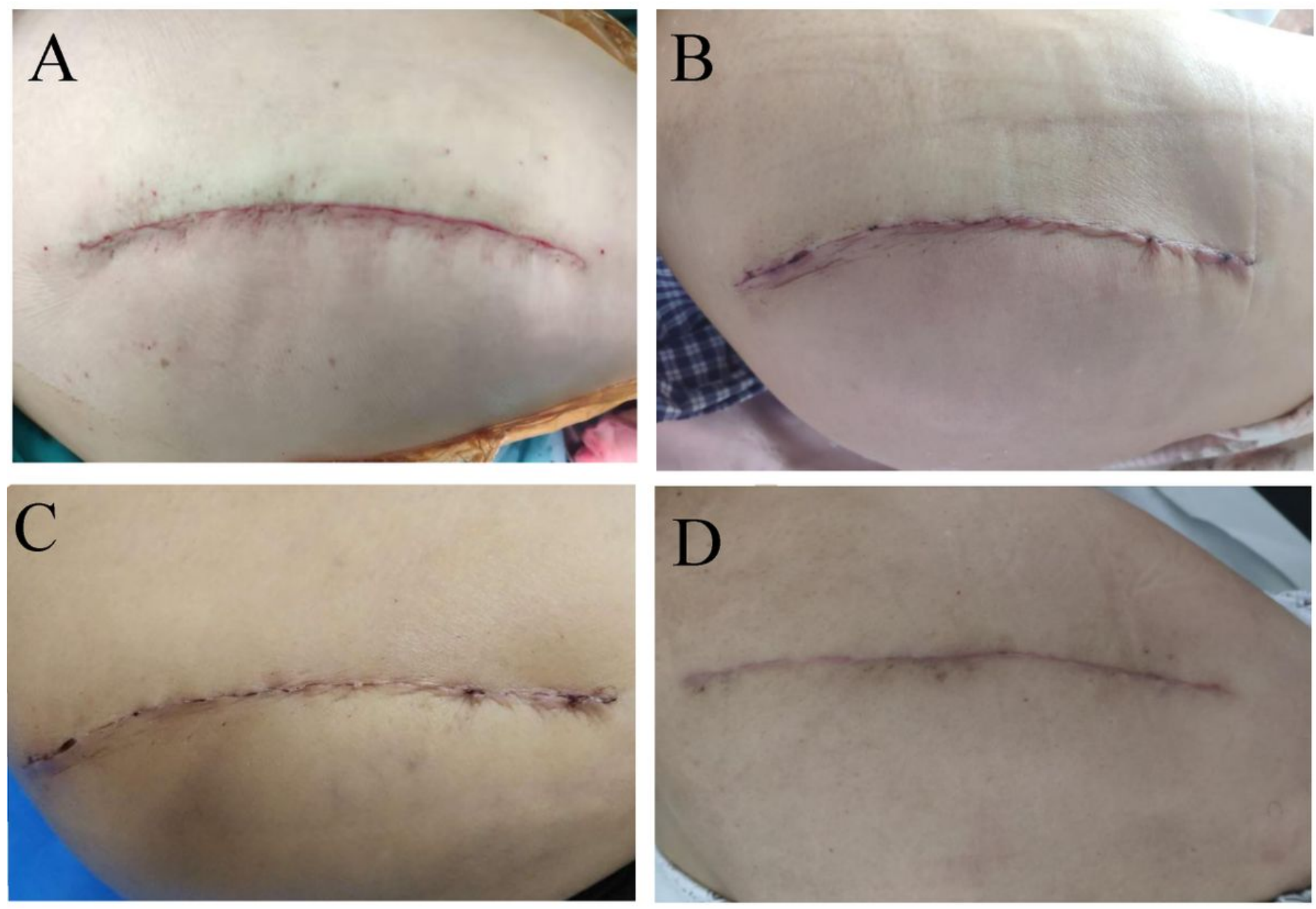

\section{Figure 1}

A. The wound was sutured during the operation. B. Three days after operation, there was no obvious ecchymosis, swelling and exudation in the wound. C. Seven days after operation, the wound healed completely. D. One month after operation, the wound of the patient showed that the scar was smooth, and the overall appearance was satisfactory. 

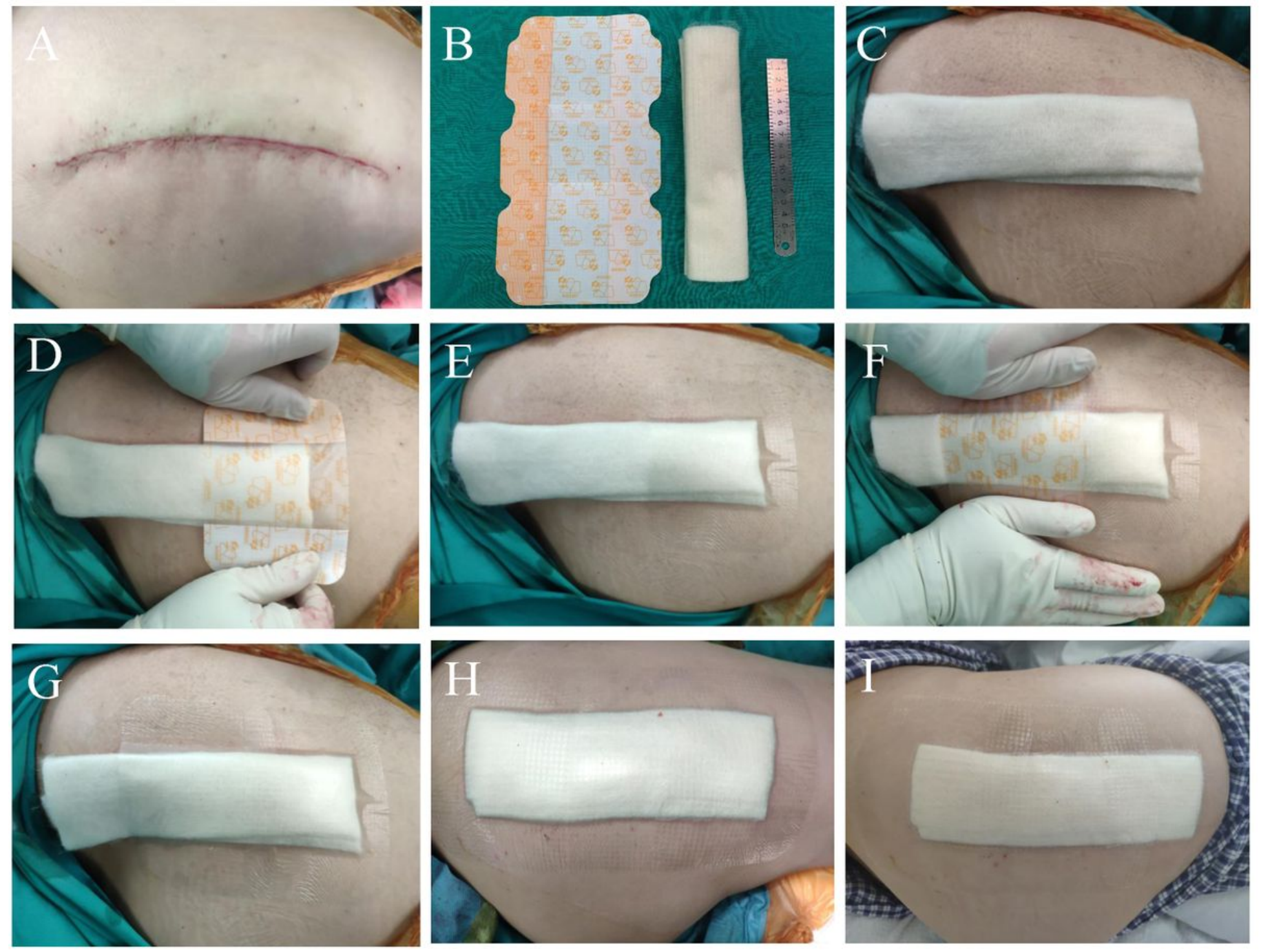

\section{Figure 1}

A. The wound was sutured and deiodized. B. Folded calcium alginate dressing and three IV3000 films. C. Cut the calcium alginate dressing to both ends slightly longer than the incision $1 \mathrm{~cm}$. D-H. According to the length of the incision, three IV3000 films were selected and applied in the order from the distal end to the proximal end of the limb. The two ends of the film were slightly longer than the incision about $4 \mathrm{~cm}$, and the latter film was overlapped and the previous one was about $1 \mathrm{~cm}$. There are no air bubbles between the films, and the skin and stick closely to the skin. I. After the patient took a bath according to his own habits, the dressing was not affected. 